\title{
Comprehensive assessment of left anterior descending coronary artery to pulmonary artery fistula
}

\author{
Alessandro Campari ${ }^{1}$, Silvia Tresoldi ${ }^{2}$, Stefano Lucreziotti ${ }^{3}$, Marco Centola ${ }^{3}$, Gianpaolo \\ Cornalba 2,4
}

1. Scuola di Specializzazione in Radiodiagnostica, Università degli Studi di Milano, Milan, Italy. 2. Department of Diagnostic and Interventional Radiology, A.O. San Paolo, Milan, Italy. 3. Department of Cardiology, A.O. San Paolo, Milan, Italy. 4. Dipartimento di Scienze della Salute, Università degli Studi di Milano, Milan, Italy.

Correspondence: Alessandro Campari. Address: Diagnostic and Interventional Radiology, S. Paolo Hospital, via A. Di Rudinì, Milano, Italy. Email: alessandro.campari@unimi.it

Received: January 19, $2014 \quad$ Accepted: February 25, $2014 \quad$ Online Published: April 11, 2014

DOI : 10.5430/ijdi.v1n2p39 URL: http://dx.doi.org/10.5430/ijdi.v1n2p39

\section{Abstract}

A 66-year-old man was hospitalized because of ST elevation myocardial infarction (STEMI). During stent placement in the medium tract of the circumflex artery, conventional coronary angiography (CCA) depicted a fistulous tract between the left anterior descending artery (LAD) and the pulmonary trunk. After exclusion of coronary steal in the LAD territories by means of rest-stress myocardial perfusion scintigraphy (MPS) with Tc-99m, one-year follow up was established. Computed tomography coronary angiography (CTCA) was performed in order to better delineate fistula's feature and provide a starting point for periodic evaluation. CTCA is less-invasive and more reproducible than conventional coronary angiography (CCA), therefore we suggest it as the modality of choice in the follow-up of coronary artery fistulas in asymptomatic patients.

\section{Keywords}

Coronary artery fistula, Conventional coronary angiography (CCA), Computed tomography coronary angiography (CTCA)

\section{Introduction}

Coronary artery fistulas are rare conditions of anomalous coronary arteries termination, in most cases incidentally identified in asymptomatic patients. The left coronary artery is slightly less commonly involved than the right. Multiple fistulas have been reported, but most are single communications, usually draining into right cardiac chambers. Natural history of coronary fistulas is variable; treatment (surgical ligation or trans-arterial embolization) is recommended in symptomatic patients or when a significant left-to-right shunt is assessed. Due to the complex 3D features of coronary artery fistulas, arteriography may incompletely delineate their course, therefore CTCA is the diagnostic modality of choice in the evaluation of these anomalies, allowing an excellent non-invasive assessment of fistulous tracts by means of multiplanar and volume rendered reformations. CTCA allows an accurate pre-operative planning, either in interventional or surgical approach. We report a case of LAD to pulmonary artery fistula studied with both CCA and CTCA. 


\section{Case presentation}

A 66-year-old man with hypertension and type II diabetes mellitus was hospitalized because of acute chest pain radiating to the left shoulder. ST tract elevation myocardial infarction (STEMI) was diagnosed. Emergency conventional coronary angiography (CCA) demonstrated the occlusion of the medium tract of the circumflex artery (CX), that was treated placing a $2.5 \mathrm{~mm} \times 16 \mathrm{~mm}$ drug eluting stent (arrow in Figure 1). Meanwhile, CCA detected an anomalous vessel originating from the left anterior descending coronary artery (LAD), consistent with coronary artery fistula, which released a high flow jet (arrowhead in Figure 1) in a lower pressure structure, most likely corresponding to the pulmonary artery. In order to rule out coronary steal due to left-to-right shunt, patient underwent rest-stress myocardial perfusion scintigraphy (MPS) with Tc-99m, which revealed hypokinesia and severe perfusion defect in the basal-inferior and mid-inferior segments and excluded inducible myocardial ischemia in the territories of the LAD. Furthermore, echocardiography revealed normal right ventricular volumes and function [Tricuspid Annular Plane Systolic Excursion (TAPSE) $=23 \mathrm{~mm}$ ]. After improvement of patient's clinical conditions, ECG-gated computed tomography coronary angiography (CTCA) was performed in order to confirm and better evaluate the fistula. A tortuous enhancing tract was detected, extending from the medium tract of the LAD to the pulmonary trunk about one centimeter above the anterior cusp of the pulmonary artery valve (arrowheads in Figure 2 and 3). Considering the MPS results and the absence of symptoms, conservative management was planned. At one year follow-up patient's conditions were stable: no symptoms were reported and dipyridamole stress echocardiography confirmed the absence of stress-induced ischemia. CECT (see Figure 3) demostrated fistula's stability, being its caliber and extent unchanged, together with stent patency.

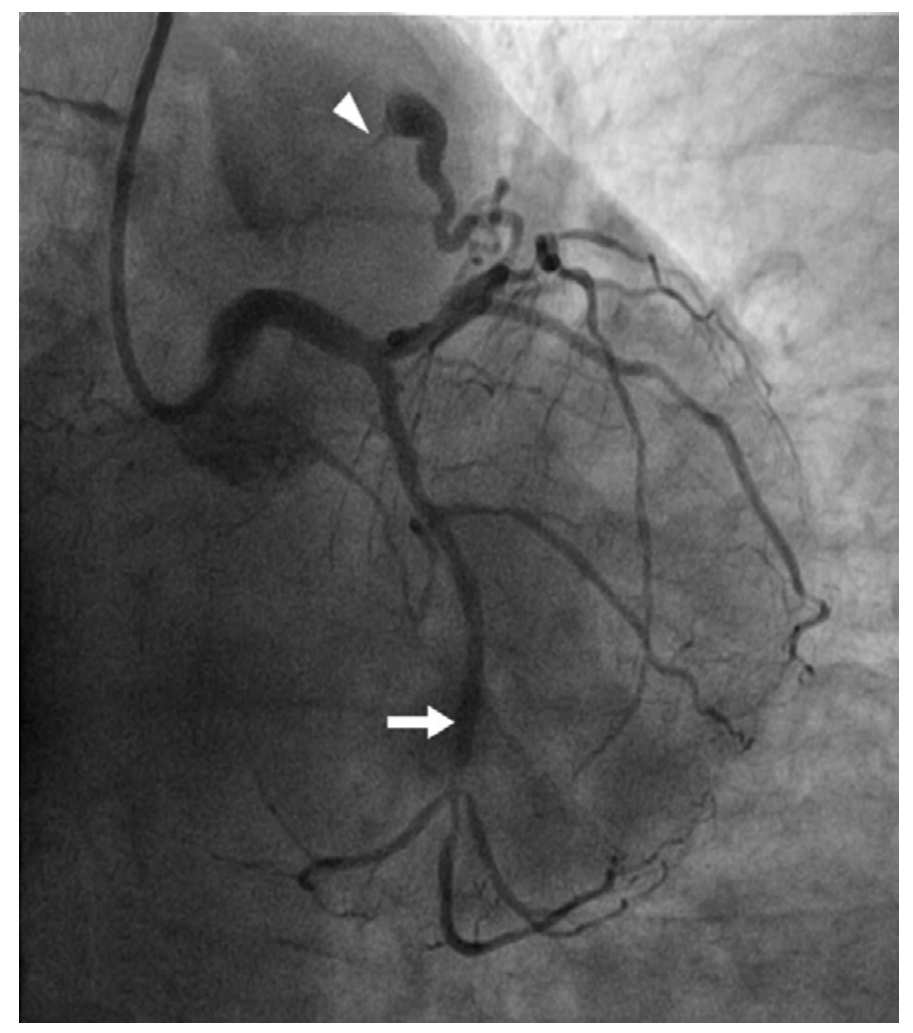

Figure 1. Conventional coronary angiography (CCA) performed after stent placement on the medium tract of the circumflex artery (arrow), shows an anomalous vessel originating from the left anterior descending coronary artery, releasing a high flow jet (arrowhead) in a lower pressure structure. 


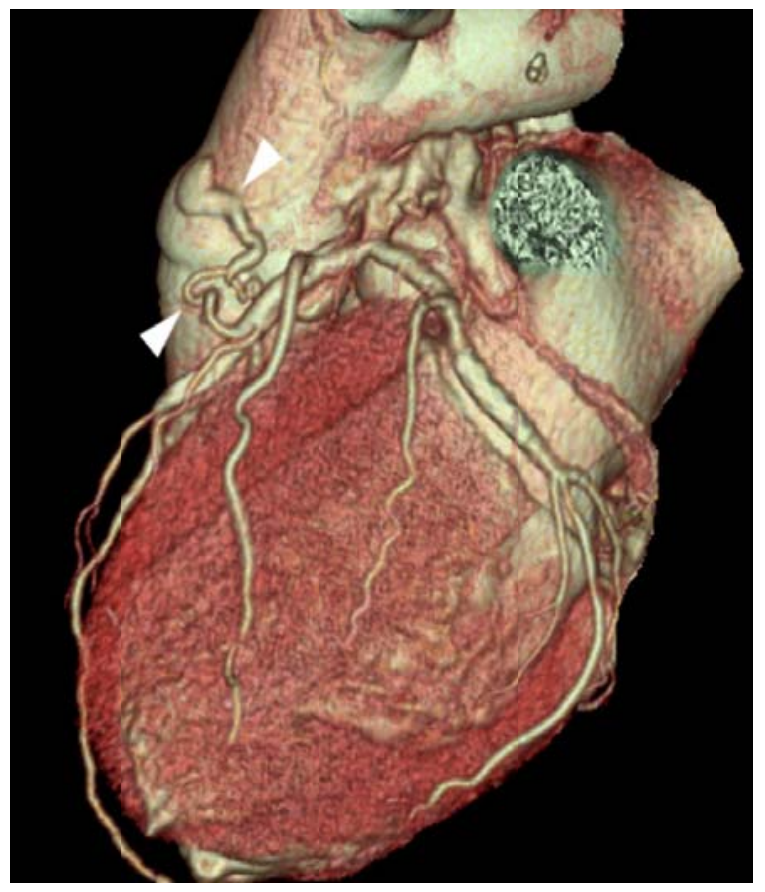

Figure 2. 3D reconstruction from CTCA dataset allows an accurate assessment of the fistula (arrowheads), extending from the medium tract of the LAD to the pulmonary trunk.

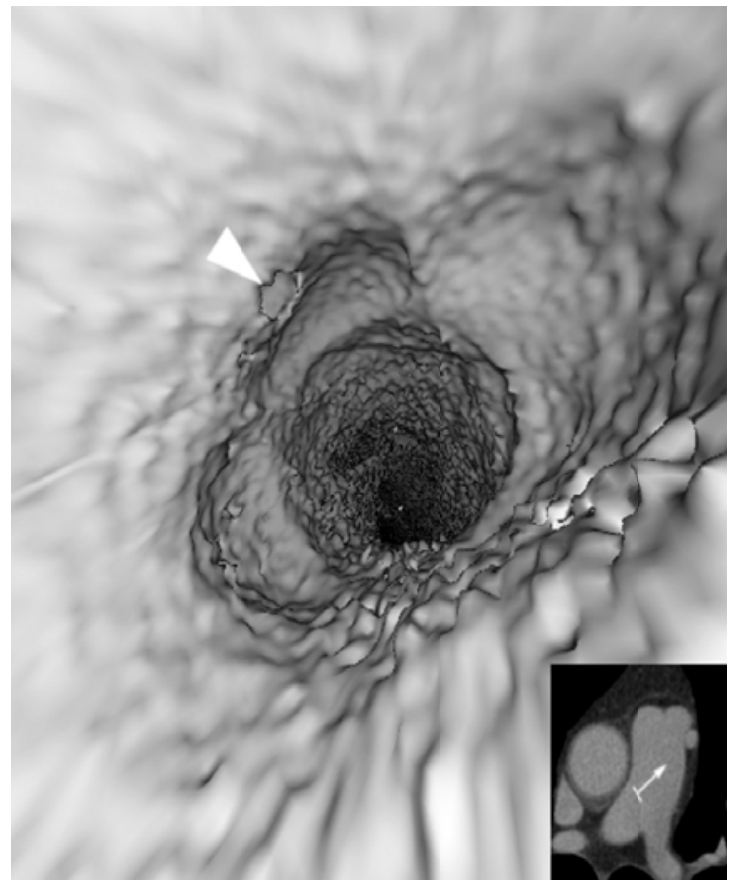

Figure 3. "Virtual Angiography" reformatted image from CTCA dataset depict the distal orifice of the fistulous tract (arrowhead) situated one cm above the anterior cusp of the pulmonary artery valve.

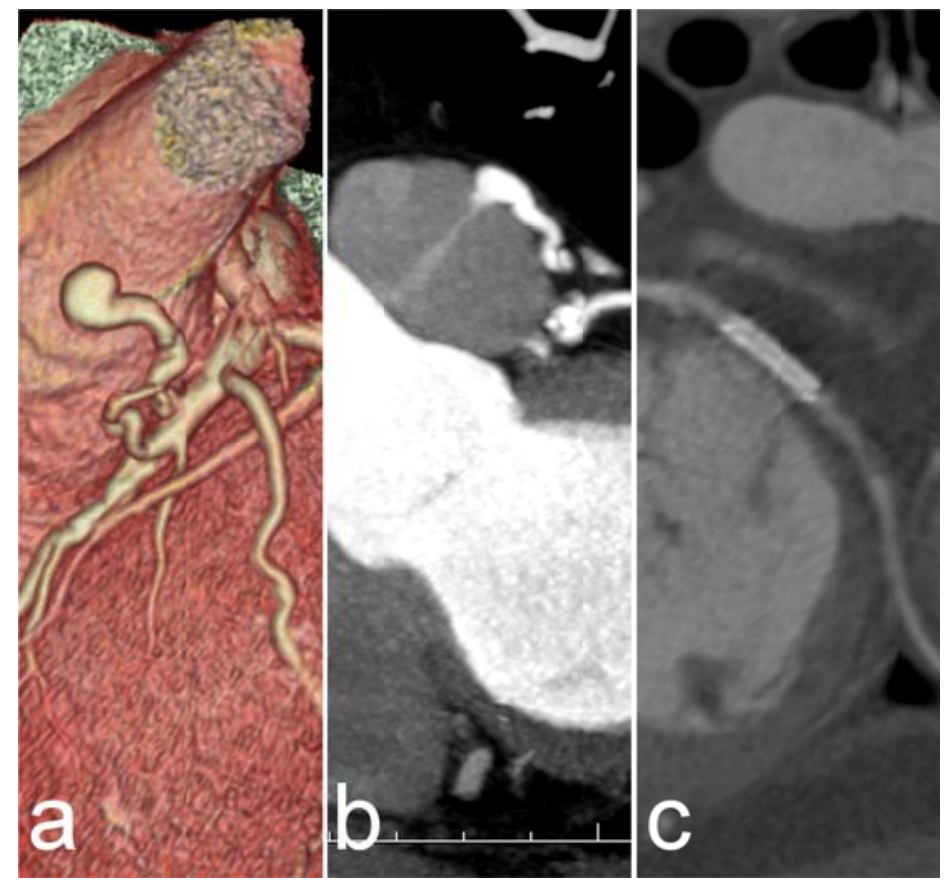

Figure 4. One year follow-up CTCA. 3D reconstruction (a) shows that fistula's features are unchanged. Multiplanar reformatted image (b) depicts the high flow jet into pulmonary artery as shown in CCA (arrowhead in Figure 1). Curved reformatted image demonstrates patency of the stent placed in CX. 


\section{Discussion}

Coronary artery fistulas are direct pre-capillary communications between one or more coronary arteries and either a cardiac chamber or other vessels. They are often due to abnormal embryological development, but may also be acquired from trauma or invasive cardiac procedures. In the United States, coronary artery anomalies are seen in 0.3 to $1.3 \%$ of patients undergoing diagnostic coronary angiography, in about 1\% of routine autopsy examinations, and in 4 to $15 \%$ of young individuals who experience sudden death ${ }^{[1]}$. The right coronary artery (RCA) is more commonly involved than the left, with an estimated prevalence rate of $60 \%$ and $40 \%$ respectively. Common drainage sites are the right ventricle (45\%), the right atrium (25\%) and pulmonary arteries (15\%). Multiple fistulas have been reported, but most are single communications. The termination of the fistulous tract has greater physiologic importance than the source: a communication with a right cardiac chamber causes a left-to-right shunt, while a drainage into a left one can mimic aortic insufficiency. Furthermore, the portion of the myocardium supplied by the involved coronary artery can undergo ischemic damage because of hemodynamic steal. A left-to-right shunt exists in over $90 \%$ of cases, however, only major fistulas cause significant blood flow from coronary system to lower pressure structures, though, in most cases patients are asymptomatic. In symptomatic patients clinical presentations include dyspnea, fatigue, chest pain and less frequently endocarditis, pericardial effusion or sudden death. The commonest clinical sign is a continuous heart murmur ${ }^{[2]}$. The majority of coronary artery fistulas are accidentally found during diagnostic investigations with CCA or CTCA as tortuous communications originating from dilated coronary artery because of increased blood flow. CCA confidently depict origin, size and number of fistulas. Yet, fistulas are heterogeneous at their distal aspect, where multiple communications or a diffuse network of vessel can be present. Moreover, when they drain into lower pressure structures, significant dilution of contrast medium occurs. In such cases, CCA may not well delineate the entire extent of the fistula ${ }^{[3,4]}$. CTCA is a well-proven non-invasive imaging modality that provides an excellent overview of the cardiac vascular anatomy and high-resolution imaging of both origin and termination of fistulous tracts, allowing its complete depiction. Compared with CCA, CTCA can provide a more accurate assessment of coronary fistulas in less time, administering less contrast medium and avoiding burden and complications of arterial puncture and vascular catheterization ${ }^{[5]}$. On the other hand, indisputable advantage of CCA is the opportunity to perform intra-operative therapeutic maneuvers. Natural history of coronary fistulas is variable. Although uncommon, spontaneous closure of the fistula has been reported. The presence of symptoms or a significant left-to-right shunt are the main indications for treatment. Available options are surgical ligation and transarterial embolization, both having similar early effectiveness, morbidity and mortality ${ }^{[6]}$. CT allows an accurate pre-operative planning, either in interventional or surgical approach. In asymptomatic adult patients close follow-up, antiplatelet therapy and preventive therapy against bacterial endocarditis is recommended ${ }^{[2,7]}$. In our case, the patient was asymptomatic and provocative MPS demonstrated absence of downstream hemodynamic steal, therefore a conservative management with one year follow-up was established. Because of fast, non-invasive and reproducible imaging, and simultaneous evaluation of both fistulous tract and stent patency, CTCA has been considered the best imaging modality for the periodic evaluation of the fistula.

\section{References}

[1] Kim SY, Seo JB, Do KH, et al. Coronary artery anomalies: classification and ECG-gated multi-detector row CT findings with angiographic correlation. Radiographics. 2006; 26: 317-333. http://dx.doi.org/10.1148/rg.262055068

[2] Gowda RM, Vasavada BC, Khan IA. Coronary artery fistulas: clinical and therapeutic considerations. Int J Cardiol. 2006; $107:$ 7-10. http://dx.doi.org/10.1016/j.ijcard.2005.01.067

[3] Zeina AR, Blinder J, Sharif D, Rosenschein U, Barmeir E. Congenital coronary artery anomalies in adults: non-invasive assessment with multidetector CT. Br J Radiol. 2009; 82: 254-261. http://dx.doi.org/10.1259/bjr/80369775

[4] Zenooz NA, Habibi R, Mammen L, Finn JP, Gilkeson RC. Coronary artery fistulas: CT findings. Radiographics. 2009; 29: 781-789. http://dx.doi.org/10.1148/rg.293085120

[5] Tresoldi S, Mezzanzanica M, Campari A, Salerno Uriarte D, Cornalba G. Role of computed tomography coronary angiography in the management of coronary anomalies. J Card Surg. 2013; 28(1): 33-6. http://dx.doi.org/10.1111/jocs.12040

[6] Armsby LR, Keane JF, Sherwood MC, Forbess JM, Perry SB, Lock JE. Management of coronary artery fistulae. Patient selection and results of transcatheter closure. J Am Coll Cardiol. 2002; 39: 1026-1032. http://dx.doi.org/10.1016/S0735-1097(02)01742-4

[7] Abdelmoneim SS, Mookadam F, Moustafa S, et al. Coronary artery fistula: single-center experience spanning 17 years. J Interv Cardiol. 2007; 20: 265-274. http://dx.doi.org/10.1111/j.1540-8183.2007.00267.x 